Editorial

\title{
Sleep re-regulation can accelerate aphasia therapy: a perspective
}

Keywords: Stroke; Attention; Neural plasticity; Sleep deprivation; Aphasia

\section{Editorial}

Stroke as the most common vascular disorder is considered the second cause of death through the world in which it is resulted in the cause of different disabilities in the adults. ${ }^{1}$ Moreover, sleep is affected subsequent to lesions affecting the central nervous system such as stroke. Also, sleep disorders can interfere in the outcome and recovery of stroke patients. ${ }^{2}$

Functionally, obviously sleep is associated to cognition structures namely; memory, attention, executive function, learning and, in general, to the mechanisms of neural plasticity, and naturally cognitive impairments are one of the main consequences of sleep deprivation..$^{3,4}$

Many studies have shown strict relationship between neural plasticity and sleep deprivation in which long sleep loss can jeopardize the execution of neurocognitive and behavioral processes. Then, this fact is worse when one encounters with brain damage. Then, if sleep plays a role in modulating cortical plasticity, rehabilitative programs and techniques should be designed considering how sleep could improve recovery. ${ }^{5,6}$

One of the consequences following stroke is speech and language disorders that is called aphasia. Aphasia co-occurs with sleep disturbances like sleep apnea and it is suggested accompanying with speech therapy clinicians consider sleep treatment that it may hold the key for improved speech therapy outcomes.

\section{Acknowledgements}

None.
Volume 2 Issue I - 2018

\begin{abstract}
Ahmad R Khatoonabadi
Department of speech therapy, Tehran University of Medical Sciences, Iran

Correspondence: Ahmad R Khatoonabadi, Department of speech therapy, Tehran University of Medical Sciences, Iran, Email Khatoonabadi@tums.ac.ir

Received: February 11, 2018 | Published: February 19,
2018
\end{abstract}

\section{Conflicts of interest}

The author declares o conflict of interest.

\section{References}

1. Bonita R. Epidemiology of stroke. Lancet. 1992;339(8789):342-344.

2. Ferre A, Ribó M, Rodríguez-Luna D, et al. Strokes and their relationship with sleep and sleep disorders. Neurologia. 2013;28(2):103-118.

3. Walker MP. Cognitive consequences of sleep and sleep loss. Sleep Medicine. 2008;9(1):S29-34.

4. McCoy JG, Strecker RE. The cognitive cost of sleep lost. Neurobiol Learn Mem. 2011;96(4):564-582.

5. Bonnet MH, Arand DL. Clinical effects of sleep fragmentation versus sleep deprivation. Sleep Med Rev. 2003;7(4):297-310.

6. Gorgoni M, D'Atri A, Lauri G, et al. Is sleep essential for neural plasticity in humans, and how does it affect motor and cognitive recovery? Neural Plast. 2013;2013:103949. 